\title{
Experimental validation of a finite difference algorithm to simulate Breathing Wall components
}

\author{
Andrea Alongi ${ }^{1}$, Adriana Angelotti ${ }^{1}$, Livio Mazzarella ${ }^{1}$ \\ ${ }^{1}$ Politecnico di Milano, Milano, Italy
}

\begin{abstract}
In this work, a one-dimensional Finite Difference Model for Breathing Wall components under time dependent Dirichlet boundary conditions is presented. This algorithm is experimentally validated at various airflow velocities, using temperature distributions and heat flux densities data collected through a set of test performed on a no-fines concrete sample in the Dual Air Vented Thermal Box (DAVTB facility).

At the end of the validation process, along with a sensitivity analysis, a good performance of the model is found in predicting the temperature distribution, with an average error calculated for each test from $0.07^{\circ} \mathrm{C}$ to $0.10^{\circ} \mathrm{C}$, and standard deviation from $0.04{ }^{\circ} \mathrm{C}$ to $0.07^{\circ} \mathrm{C}$. At the same time, the heat flux density prediction is proven to be highly dependent on the evaluation of the material thermal-physical properties and on the measurement of temperature boundary conditions.
\end{abstract}

\section{Introduction}

Dynamic Insulation, also known as Breathing Wall, is a building envelope technology based on air permeable components linked to the ventilation system and crossed by a desired airflow. Hence, external walls and roofs perform as heat exchangers (Taylor et al., 1996; Taylor and Imbabi,1998; Imbabi, 2006; Craig and Grinham, 2017) and filters (Taylor et al., 1999; Imbabi, 2004).

In the past, this technology has been mostly investigated in steady state conditions with air moving inward and heat flux moving outward (namely, contra flux condition), both numerically (Imbabi, 2012; Gan, 2000) and experimentally (Dimoudi, 2004; Imbabi, 2006; Di Giuseppe, 2015).The analytical model that predicts the behavior of Breathing Walls in steady state conditions, established by Taylor, has been experimentally validated by the Authors (Alongi et al., 2017a; Alongi et al., 2017b) on a small sample of no-fines concrete (Wong, 2007).

As far as unsteady conditions are concerned, an analytical model for steady periodic third type conditions can be found in literature (Krarti, 1994). However, in order to analyse the energy performance of a Breathing Wall in a building subjected to outside weather and internal gains, a dynamic model able to describe any variable regime is necessary. This leads to develop numerical models for heat transfer across Breathing Walls in variable regime.

A one-dimensional Finite Difference model based on the Crank-Nicholson approach is presented in (Ascione et al.,
2015), where the validation process is performed using both the steady state Taylor model and a commercial FEM software for the transient conditions.

In this work, a full implicit one-dimensional Finite Difference Model for Breathing Wall technologies is presented and experimentally validated. Validation is performed through direct comparisons with temperature distribution and heat flux density measurements collected on a no-fines concrete sample subjected to steady periodic boundary conditions. A sensitivity analysis is provided, to evaluate the effects of time and space discretizations in the numerical scheme. Finally, the consequences of uncertainties in the measurement of material properties and boundary conditions are discussed.

\section{Methods}

The Breathing Wall unsteady heat transfer model is presented below, along with the numerical model, experimental setup and wall sample.

\section{The physical model}

The whole section of a Breathing Wall is crossed by a conductive flux, caused by the indoor-outdoor temperature difference, and an advective one, generated by the imposed airflow. If we introduce the hypothesis of homogeneous and isotropic medium and one dimensional heat flux, the heat transfer phenomenon can be described through a modified Fourier equation such as:

$$
(\rho c)_{w} \frac{\partial T}{\partial t}+u_{f}\left(\rho c_{p}\right)_{f} \frac{\partial T}{\partial x}=\lambda_{w} \frac{\partial^{2} T}{\partial x^{2}}
$$

where $T$ is the temperature distribution in the domain, assumed as a function of time $t$ and space $x$, and $\rho, c$ and $\lambda$ are the density, the specific heat (at constant pressure if $p$ subscript is indicated) and the thermal conductivity of porous material (subscript $w$ ) or air (subscript $f$ ). Going more in detail about the domain, this work is focused on a single layer component, with the spatial coordinate from the outer surface $(x=0)$ to the inner one $(x=L)$.

Usually, third type boundary conditions are combined with Eq. (1): namely, the conductive heat flux on the edge of the domain is assumed to be equal to a convectiveradiative one on the same side, calculated as a function of an operative temperature for the environment and a heat transfer coefficient. However, in this work, in order to simplify the validation process, first type boundary conditions are considered and surface temperature values are imposed. More details are given in the following sections. The equations for the boundary conditions are: 


$$
\begin{array}{ll}
\text { outer surface } & T(0, t)=T_{0}(t) \\
\text { inner surface } & T(L, t)=T_{L}(t)
\end{array}
$$

\section{The numerical model}

In order to be able to assess the thermal behaviour of Breathing Wall components and calculate the temperature distribution across their section for a given set of timedependent Dirichlet boundary conditions, a onedimension numerical algorithm has been written in Matlab ${ }^{\circledR}$, and the differential problem represented by Eq. (1), (2) and (3) has been studied using a Finite Difference Method. The central difference scheme has been used to approximate both spatial derivatives (second order for the diffusive term and first order for the advective one), while the time variation has been managed using the fully implicit representation (backward Euler).

Dividing Eq. (1) by the wall thermal capacity $(\rho c)_{w}$, all the thermal-physical properties can be summarized into:

$$
\begin{aligned}
& \alpha=\frac{\lambda_{w}}{(\rho c)_{w}} \\
& \beta=u_{f} \frac{\left(\rho c_{p}\right)_{f}}{(\rho c)_{w}}
\end{aligned}
$$

namely, the porous medium thermal diffusivity $(\alpha)$ and the fluid revised velocity $(\beta)$. Moreover, the spatial domain is discretized into a structured grid with a $\Delta x$ step, while the temporal dimension is divided into $\Delta t$ timesteps. If the space domain is divided into $N+1$ nodes and the time domain into $M+1$ nodes, the discrete field equation referred to the $i$-th node at the $j$-th timestep becomes:

$$
\begin{gathered}
\frac{T_{i}^{j+1}-T_{i}^{j}}{\Delta t}+\beta \frac{T_{i+1}^{j+1}-T_{i-1}^{j+1}}{2 \Delta x}=\alpha \frac{T_{i+1}^{j+1}-2 T_{i}^{j+1}+T_{i-1}^{j+1}}{\Delta x^{2}} \\
\text { with }\left\{\begin{array}{l}
i=1 \div N \\
j=0 \div M
\end{array}\right.
\end{gathered}
$$

Eq. (6) leads then to the following numerical scheme:

$$
\begin{aligned}
& T_{i+1}^{j+1}\left(\frac{\beta \Delta t}{2 \Delta x}-\frac{\alpha \Delta t}{\Delta x^{2}}\right)+ \\
& +T_{i}^{j+1}\left(1+2 \frac{\alpha \Delta t}{\Delta x^{2}}\right)+ \\
& +T_{i-1}^{j+1}\left(-\frac{\beta \Delta t}{2 \Delta x}-\frac{\alpha \Delta t}{\Delta x^{2}}\right)=T_{i}^{j}
\end{aligned}
$$

For this work, boundary conditions are defined as first type and feature steady periodic trend. Initial condition is a linear temperature distribution across the domain, based on the initial values of the boundary conditions:

$$
\begin{array}{ll}
T_{0}^{j}=\bar{T}_{0}+\Delta T_{0} \sin \left(\omega j \Delta t+\psi_{0}\right) & \text { at } x=0 \\
T_{N}^{j}=\bar{T}_{N}+\Delta T_{N} \sin \left(\omega j \Delta t+\psi_{L}\right) & \text { at } x=N \Delta x=L \\
T_{i}^{0}=\frac{T_{N}^{0}-T_{0}^{0}}{L} i \Delta x+T_{0}^{0} & \text { at } t=0
\end{array}
$$

where $\bar{T}$ is the stationary component of the surface temperature, $\Delta T$ its fluctuation, $\omega$ and $\psi$ are the angular frequency and phase of the sinusoidal component respectively. The Matlab ${ }^{\circledR}$ script is potentially able to deal with multi-layer components and third type boundary conditions, commonly used in building simulations. However, due to the complexity in measuring heat transfer coefficients, the validation process is performed on the Dirichlet boundary conditions only.
Finally, the temperature distribution obtained at each time step has been used to calculate the corresponding heat flux density at the $x=L$ surface, defined as:

$$
\varphi_{L}^{j}=-\lambda_{w} \frac{T_{N}^{j}-T_{N-1}^{j}}{\Delta x}
$$

\section{The experimental setup}

The numerical model experimental validation has been performed through direct comparison with temperature distribution measurements collected using a laboratory rig called Dual Air Vented Thermal Box (DAVTB). This setup is able to test small scale samples of building envelope technologies under user-defined thermal boundary conditions (either steady state or steady periodic) and force a controlled airflow through permeable components (e.g. Breathing Walls). A detailed description is provided in previous works by the Authors (Alongi and Mazzarella, 2015; Alongi et al., 2017a).

The facility (Figure 1) consists of two insulated chambers Box 1 and Box 2 (each $1.5 \mathrm{~m}$ wide $x 1.5 \mathrm{~m}$ high $\mathrm{x} 1.29 \mathrm{~m}$ long) separated by the $1.5 \mathrm{~m} \times 1.5 \mathrm{~m}$ insulated metal frame that accommodates the sample. Moreover, they are externally connected by the air recirculation system, used to generate an airflow through the sample itself. The setpoint operative temperature is defined separately in each chamber in a range between $15^{\circ} \mathrm{C}$ and $50^{\circ} \mathrm{C}$, and is achieved by means of a dedicated hydronic system providing both heating and cooling power through radiant panels inside the two boxes.

The measurement and control system in the DAVTB apparatus is based on an Agilent 34980A multifunctional switch unit, remotely controlled through a LabVIEW algorithm. Temperature measurements are performed in several points of the hydraulic plant, in various locations inside each chamber and inside the sample wall, using Ttype calibrated thermocouples (TC). Moreover, a globe thermometer is installed in the geometrical center of each chamber to measure the operative temperature. Following the calibration process, for all temperature probes an accuracy of $0.15^{\circ} \mathrm{C}$ has been considered. Finally, a $4.4 \mathrm{~mm}$ x $4.4 \mathrm{~mm}$ heat flux meter (gSKIN ${ }^{\circledR}-\mathrm{XM} 26$ 9C) has been installed on the sample surface facing Box 2, and features a $\pm 3 \%$ calibration accuracy according to the manufacturer GreenTeg.

The average air velocity across the sample is measured through a bi-directional fan anemometer located in a dedicated section of the air recirculation pipe. The anemometer, a vane wheel sensor produced by Hoentzsch $\mathrm{GmbH}$, operates in the range $\pm(0.4 \div 20) \mathrm{m} / \mathrm{s}$, and the accuracy of the overall measurement chain has been assessed at $\pm 1.3 \cdot 10^{-4} \mathrm{~m} / \mathrm{s}$.

As far as the sample is concerned, in this work the analysis has been focused on a $0.15 \mathrm{~m}$ thick single layer Breathing Wall component based on no-fines concrete. The wall is also divided into nine $0.32 \mathrm{~m} \times 0.32 \mathrm{~m}$ blocks and thermocouples are embedded in the centermost section, with nine probes displaced at $1.5 \mathrm{~cm}$ apart from each other and two more to measure the surface temperatures (Figure 2). 


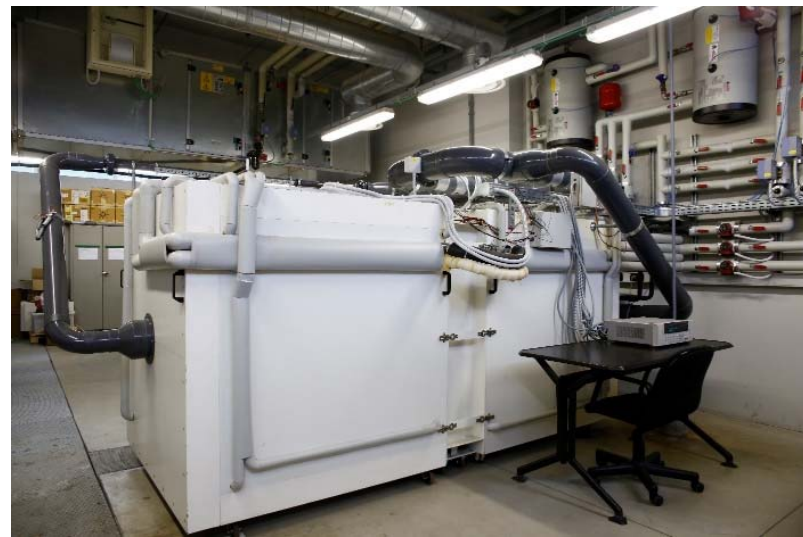

Figure 1: the DAVTB facility.

No-fines concrete consists of a cement based mixture involving large aggregates only (i.e. gravel with an average diameter in the range $6 \mathrm{~mm} \div 12 \mathrm{~mm}$ ) and features a highly interconnected porous matrix (Wong, 2007; Alongi et al., 2017a). Through Transient Plane Source technique, mass and volume measurements its thermal-physical properties have been experimentally assessed. They are listed in Table 1, along with the air properties used in this work during the simulation phase.

Table 1: thermal-physical properties of air and no-fines concrete used in this work.

\begin{tabular}{ccc}
\hline quantity & Air & no-fines \\
\hline porosity $\varepsilon$ & - & $(30 \pm 2) \%$ \\
density $\rho$ & $1.23 \mathrm{~kg} / \mathrm{m}^{3}$ & $(1738 \pm 61) \mathrm{kg} / \mathrm{m}^{3}$ \\
specific heat capacity $c$ & $1004.9 \mathrm{~J} /(\mathrm{kg} \cdot \mathrm{K})$ & $(1011 \pm 110) \mathrm{J} /(\mathrm{kg} \cdot \mathrm{K})$ \\
thermal conductivity $\lambda$ & $0.025 \mathrm{~W} /(\mathrm{m} \cdot \mathrm{K})$ & $(1.24 \pm 0.09) \mathrm{W} /(\mathrm{m} \cdot \mathrm{K})$ \\
\hline
\end{tabular}

The validation process applied to the numerical model discussed above is based on a series of tests performed imposing an operative temperature steady periodic condition in one chamber and a steady state one in the other. A $24 \mathrm{~h}$ period sinusoidal pattern is replicated in Box 1 , with average temperature $26^{\circ} \mathrm{C}$ and amplitude $6{ }^{\circ} \mathrm{C}$, representing the outdoor environment in Milan during summer. In Box 2 the operative temperature is kept stationary at $26^{\circ} \mathrm{C}$, representing an indoor summer condition for a residential or office building. As far as airflow is concerned, five different average air velocities have been tested $(0.001 \mathrm{~m} / \mathrm{s}, 0.003 \mathrm{~m} / \mathrm{s}, 0.006 \mathrm{~m} / \mathrm{s}$, $0.009 \mathrm{~m} / \mathrm{s}$ and $0.012 \mathrm{~m} / \mathrm{s}$ ), along with a reference test without airflow (airtight) to replicate the behaviour of a traditional wall as a benchmark. Data are collected every $5 \mathrm{~s}$ for a period of at least three days in order to guarantee the achievement of the desired condition, which is assessed through the observation of the measured operative temperatures.

The validation process is conducted as follows: surface temperatures measured in each test are filtered using the Matlab ${ }^{\circledR}$ Curve Fitting Toolbox, to achieve analytical equations to derive these quantities at every necessary time and to neglect any small fluctuation. The results of the fitting process have then been used as boundary conditions to run the numerical, along with the

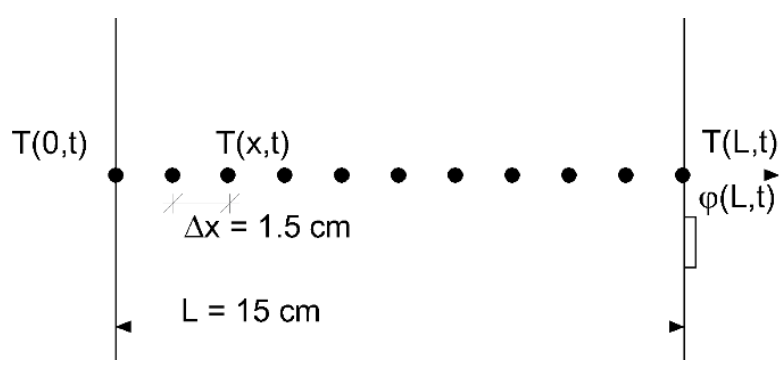

Figure 2: temperature and heat flow measurements inside and at the surfaces of the sample wall.

appropriate airflow rate value corresponding to each test. The outcomes of the numerical simulations (i.e. temperature distribution across the calculation domain and heat flux density at the inner surface) have then been directly compared to the corresponding experimental data.

\section{Results and Discussion}

The outcomes of the experimental campaign are presented and discussed. Then, the numerical results are validated in terms of temperature distribution and heat flux density.

\section{Experimental results and relative processing}

As a first step, the six experimental tests previously illustrated have been performed: temperature distributions across the section of the no-fines concrete slab have been sampled under steady periodic boundary conditions and with various levels of crossing airflow velocity.

Moreover, during each test the heat flux density on the surface facing Box 2 has been measured. Collected data have been inspected to assess the achievement of the desired experimental boundary conditions: namely, a periodically recursive fluctuation for the operative temperature in Box 1 and a stationary operative temperature in Box 2. Measurements show an average control error of $-0.05^{\circ} \mathrm{C} \div 0.04{ }^{\circ} \mathrm{C}$ with a standard deviation of $0.05{ }^{\circ} \mathrm{C} \div 0.13{ }^{\circ} \mathrm{C}$, depending on the test. Even though some discrepancies between the set-point and the actual temperatures have been observed when the system switches from heating to cooling regime and vice versa, these fluctuations are not relevant for the overall outcomes of the tests. This means that the DAVTB facility is able to achieve the desired conditions with an acceptable level of accuracy, and the surface temperature measurements, as shown in Figure 3(a) and (b), can then be used to derive the boundary conditions for the simulation process discussed below.

Surface temperatures too have been investigated to verify the achievement of the steady periodic condition, which is clearly observable in Figure 3(a) and (b). Then, these quantities have been processed using the Matlab ${ }^{\circledR}$ Curve Fitting Toolbox to obtain analitical forms usefull as boundary conditions for the numerical model. In this way, 

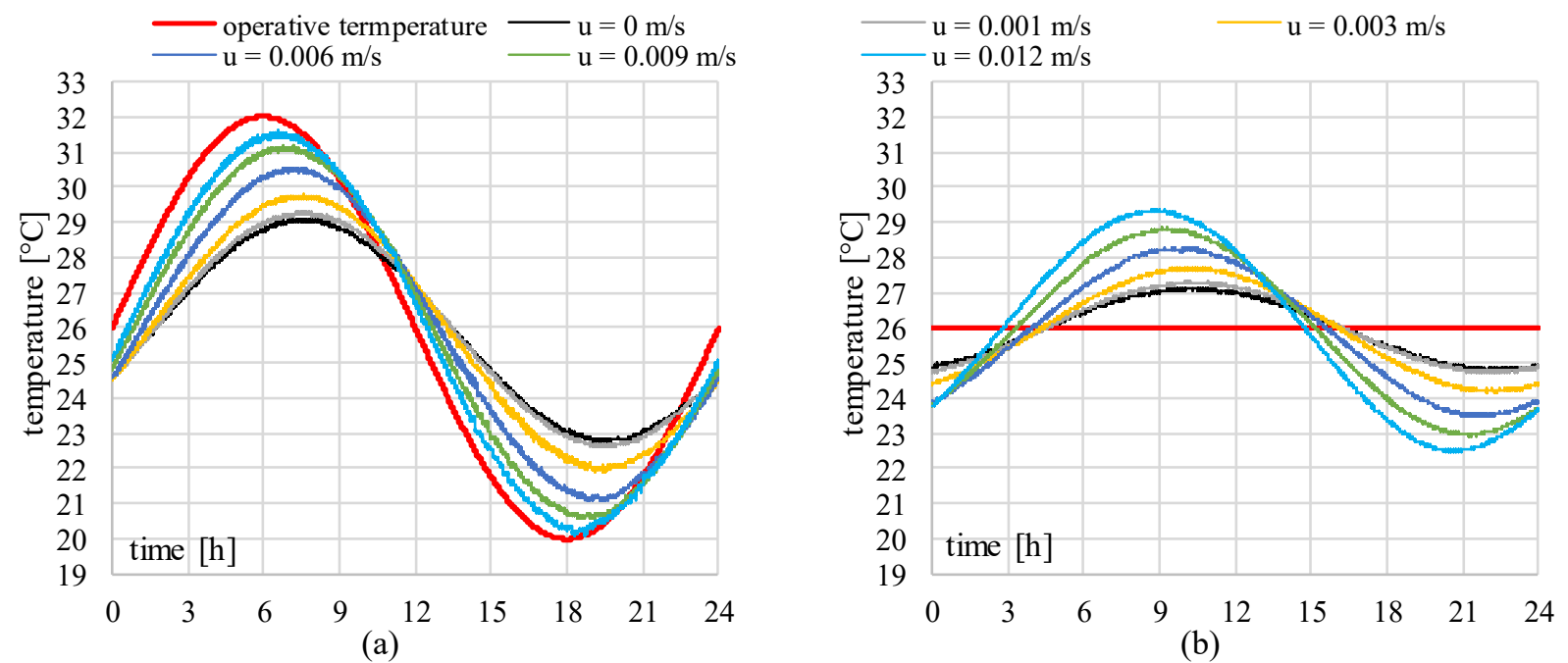

Figure 3: operative temperature (red line) and surface temperatures (other colours) measured for each test on Box 1 side (a) and on Box 2 side (b).

it has been possible to obtain the $\bar{T}, \Delta T$ and $\psi$ required in Eqs. (8) and (9), with the assumption of the angular frequency $\omega$ equal to $7.27 \cdot 10^{-5} \mathrm{rd} / \mathrm{s}$, corresponding to a period of $24 \mathrm{~h}$. The results of this process are summarized in Table 2.

Table 2: results of the fitting process applied on the surface temperatures measured on the Box $1\left(T_{0}\right)$ and on the Box $2\left(T_{N}\right)$ sides for each test performed.

\begin{tabular}{ccccccc} 
& \multicolumn{3}{c}{$\boldsymbol{T}_{\boldsymbol{0}}$ coefficients } & Eq.(8) & \multicolumn{3}{c}{$\boldsymbol{T}_{\boldsymbol{N}}$ coefficients } & Eq.(9) \\
\hline $\mathbf{u}$ & $\overline{\boldsymbol{T}}$ & $\Delta \boldsymbol{T}$ & $\boldsymbol{\psi}$ & $\overline{\boldsymbol{T}}$ & $\Delta \boldsymbol{T}$ & $\boldsymbol{\psi}$ \\
{$[\mathbf{m} / \mathbf{s}]$} & {$\left[{ }^{\circ} \mathbf{C}\right]$} & {$\left[{ }^{\circ} \mathbf{C}\right]$} & {$[\mathbf{r d}]$} & {$\left[{ }^{\circ} \mathbf{C}\right]$} & {$\left[{ }^{\circ} \mathbf{C}\right]$} & {$[\mathbf{r d}]$} \\
\hline 0 & 25.91 & 3.12 & -0.423 & 25.95 & 1.15 & -1.149 \\
0.001 & 25.94 & 3.29 & -0.429 & 26.01 & 1.27 & -1.231 \\
0.003 & 25.88 & 3.84 & -0.380 & 25.94 & 1.73 & -1.103 \\
0.006 & 25.85 & 4.65 & -0.298 & 25.88 & 2.36 & -0.994 \\
0.009 & 25.88 & 5.22 & -0.211 & 25.89 & 2.90 & -0.833 \\
0.012 & 25.89 & 5.60 & -0.152 & 25.94 & 3.38 & -0.719 \\
\hline
\end{tabular}

Through the observation of the coefficients reported in Table 2, it is possible to draw some conclusions about the experimental phase of this work. First of all, as expected, all the stationary components $\bar{T}$ are comparable to the desired value of $26^{\circ} \mathrm{C}$ on both sides of the sample, leading to an almost null stationary component of the heat flux density. Secondly, the growth of the airflow rate velocity from a test to another leads to an increased fluctuation of both surface temperatures: as a consequence of the air motion from Box 1 to Box 2 across the sample, the operative temperature variation over time inside the first box affects the no-fines concrete slab in an increasingly relevant way.

\section{Numerical simulation results}

At this point, the data collected in Table 2 have been used in Eqs. (8) and (9), and with the airflow velocity values it has been possible to replicate the experimental tests using the Finite Difference Algorithm discussed in this work. At first, every simulation has been run using the average material properties listed in Table 1, and results have been directly compared to the measured data, in order to assess the accuracy of the numerical scheme in predicting the temperature distribution and the surface heat flux density for a given set of boundary conditions. All the velocity levels have been tested; however, only the airtight, the $0.003 \mathrm{~m} / \mathrm{s}$ and the $0.012 \mathrm{~m} / \mathrm{s}$ conditions are represented as an example in the carpet plots in Figure 4, where the temperature distribution across the section ( $x$ - ordinate) is reported for a $24 \mathrm{~h}$ time period (time - abscissa).

Going more in detail, this first group of simulations has been performed assuming a spatial grid discretization $\Delta x=1 \mathrm{~mm}$ and a timestep of $\Delta t=3600 \mathrm{~s}$, the latter being typically used in annual building simulations. This first iteration of the simulation phase provides a reference condition for further analysis. The simulation errors are then calculated as the absolute value of the difference between the measured quantity, either temperature or heat flux density, and the calculated one at any given position or time. As far as heat flux densities are concerned, since measured values show high frequency fluctuations, a direct comparison between measured and calculated data would have been unpractical. Therefore, a fitting process has been applied to this quantity too. The equation is then:

$$
\varphi_{L}(t)=\bar{\varphi}_{L}+\Delta \varphi_{L} \cos \left(\omega t+\psi_{\varphi}\right)
$$

and the coefficients are reported in Table 3. Then, average $\mu$, standard deviation $\sigma$ and maximum error $e_{\max }$ are calculated for each airflow velocity considered, for all the measured temperatures and the heat flux density, as shown in Table 4.

This first set of results shows a good performance in predicting temperatures: all the average errors and their standard deviations are well within the $\pm 0.15^{\circ} \mathrm{C}$ accuracy of the thermocouples. However, numerical results for heat flux densities are less precise: considering all the airflow conditions investigated, errors are relevant if compared to the measured heat flux density fluctuation amplitude, as represented by the $\Delta \varphi_{L}$ values listed in Table 3 . 


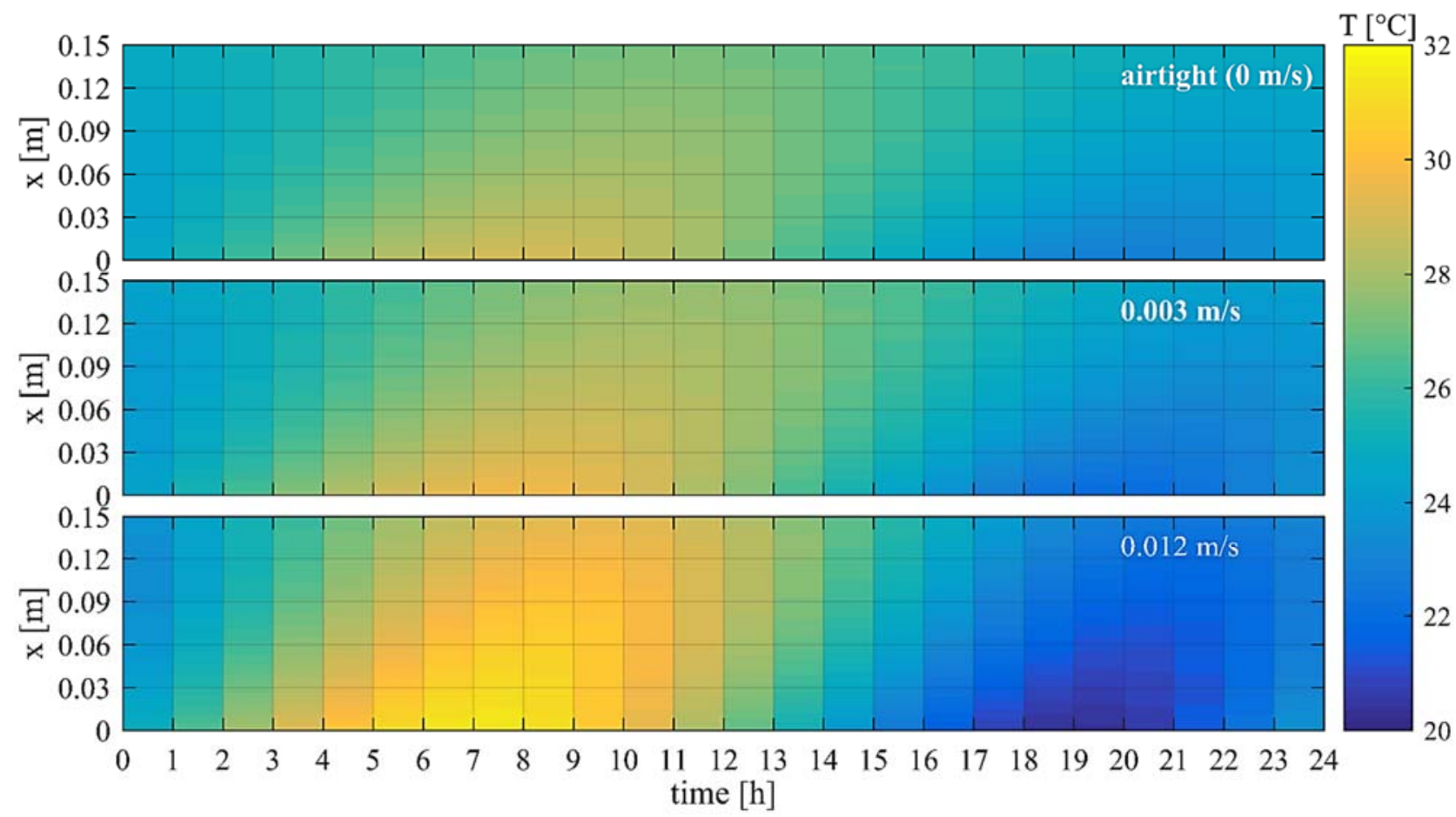

Figure 4: carpet plot of the temperature distribution across the no-fines concrete slab in a $24 \mathrm{~h}$ time period. Airtight, $0.003 \mathrm{~m} / \mathrm{s}$ and $0.012 \mathrm{~m} / \mathrm{s}$ conditions are represented.

Table 3: results of the fitting process applied on the measured heat flux densities.

\begin{tabular}{cccccccc}
\hline $\boldsymbol{u}$ & {$[\mathrm{m} / \mathbf{s}]$} & $\mathbf{0}$ & $\mathbf{0 . 0 0 1}$ & $\mathbf{0 . 0 0 3}$ & $\mathbf{0 . 0 0 6}$ & $\mathbf{0 . 0 0 9}$ & $\mathbf{0 . 0 1 2}$ \\
\hline $\bar{\varphi}_{L}$ & {$\left[\mathrm{~W} / \mathrm{m}^{2}\right]$} & -0.98 & -0.77 & -0.87 & -0.70 & -1.21 & -2.16 \\
$\Delta \varphi_{L}$ & {$\left[\mathrm{~W} / \mathrm{m}^{2}\right]$} & 13.33 & 12.49 & 14.95 & 17.45 & 20.22 & 24.98 \\
$\psi_{\varphi}$ & {$[\mathrm{rd}]$} & 0.356 & 0.413 & 0.402 & 0.493 & 0.649 & 0.773 \\
\hline
\end{tabular}

Table 4: overall average, standard deviation and maximum error calculated for temperature and heat flux density, for every airflow velocity considered.

\begin{tabular}{|c|c|c|c|c|c|c|c|}
\hline \multicolumn{2}{|c|}{$u[\mathrm{~m} / \mathrm{s}]$} & 0 & 0.001 & 0.003 & 0.006 & 0.009 & 0.012 \\
\hline \multirow{3}{*}{$\begin{array}{l}\bigcup_{0} \\
\text { E }\end{array}$} & $\mu$ & 0.07 & 0.07 & 0.07 & 0.08 & 0.09 & 0.10 \\
\hline & $\sigma$ & 0.04 & 0.04 & 0.04 & 0.05 & 0.06 & 0.07 \\
\hline & $e_{\max }$ & 0.15 & 0.19 & 0.18 & 0.19 & 0.24 & 0.26 \\
\hline \multirow{3}{*}{$\frac{\sqrt[N]{\xi}}{\sum_{a}^{E}}$} & $\mu$ & 1.90 & 1.85 & 1.89 & 2.47 & 2.43 & 2.62 \\
\hline & $\sigma$ & 1.25 & 1.27 & 1.28 & 1.28 & 1.25 & 1.22 \\
\hline & $e_{\max }$ & 4.03 & 3.88 & 3.95 & 4.21 & 4.25 & 4.36 \\
\hline
\end{tabular}

\section{Sensitivity analysis: temperatures}

A sensitivity analysis has then been performed to investigate the effects of space and time discretizations on the numerical results. First, the whole group of airflow conditions has been simulated with $\Delta t=3600 \mathrm{~s}$, halving $(0.5 \mathrm{~mm})$ and doubling $(2 \mathrm{~mm})$ the spatial grid step; then setting $1800 \mathrm{~s}$ and $900 \mathrm{~s}$ timesteps, with $\Delta x=1 \mathrm{~mm}$. The computational time is within $3.5 \mathrm{~s}$, when a time span of 10 days is simulated. This value will grow when a whole year will be considered.

Numerical results have been compared to experimental data to obtain new average, standard deviation and maximum errors. At this stage, only temperatures are considered: in Figure 5 and Figure 6 the effects of time and space discretizations are considered separately. First of all, it is possible to observe that the spatial grid dimension has not significant effects on the accuracy of the method: none of the values displayed in Figure 5 seems to be affected by a reduction or increase in $\Delta x$, possibly because all the grid dimensions considered are fine enough to achieve a set of results which is independent from the grid dimension itself. Secondly, a slight improuvement in both average and standard deviation of the simulation error is achieved with timestep progressive reduction, as shown in Figure 6, where a generally better agreement between numerical and experimental data is displayed. However, a slightly different behavior can be observed for the maximum error, which does not necessarily decrease with the reduction of the simulation timestep, especially with the highest level of advection inside the domain $(u=0.012 \mathrm{~m} / \mathrm{s})$. Yet, the difference between the maximum errors calculated at different timesteps for a given airflow condition is contained in the thermocouple accuracy of $0.15^{\circ} \mathrm{C}$; therefore, this result might be due to measurement noise and inaccuracy, rather than being a consequence of the numerical model.

Anyway, all the cases considered show average errors well within the measurement accuracy of thermocouples, and demonstrate that the numerical scheme investigated is able to reliably predict the temperature distribution across Breathing Wall components under unsteady Dirichlet boundary conditions. Moreover, it is also demonstrated that the implicit scheme adopted allows the user to assume fairly large timesteps, such as $3600 \mathrm{~s}$, which are typically adopted to reduce computational costs in building simulations.

\section{Sensitivity analysis: heat flux density}

Then, the sensitivity analysis has been focused on the ability of the numerical model to predict the heat flux density at the inner surface. 


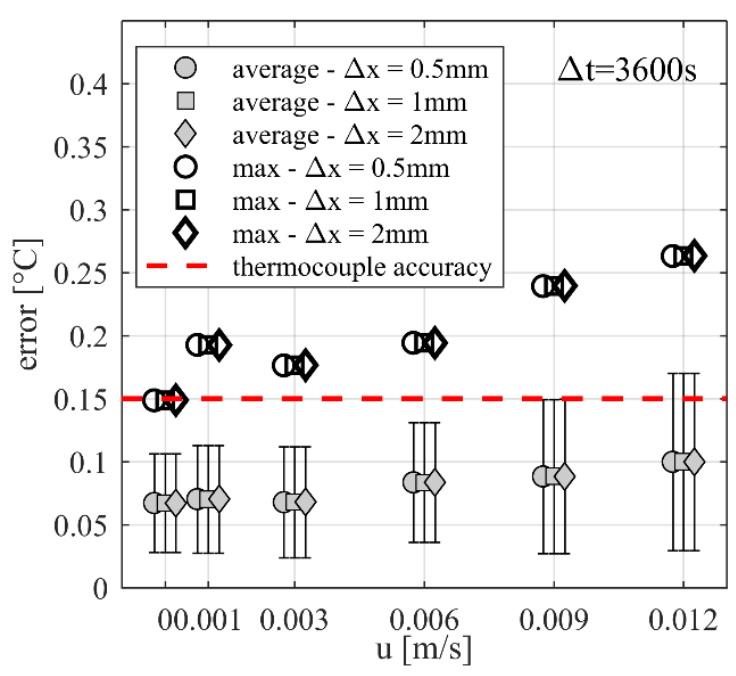

Figure 5: effects of space grid on simulation accuracy.

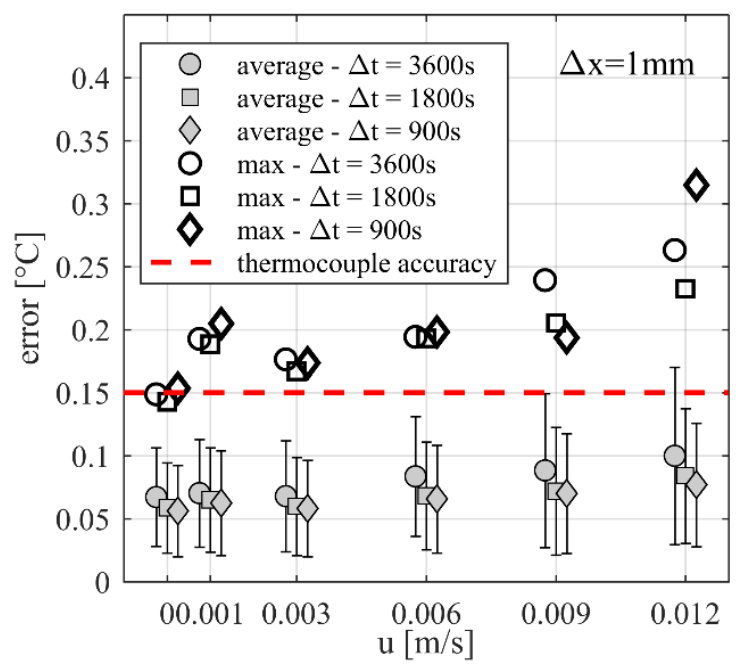

Figure 6: effects of timestep on simulation accuracy.

First of all, the effects of a variation in the material heat capacity have been calculated: it has in fact to be remembered that the no-fines concrete properties reported in Table 1 are provided in ranges, and all the simulations previously discussed have been performed considering the average values for all those quantities. In order to consider the effects of their uncertainties, all the values have been combined in order to either minimize or maximize the overall heat capacity $C=(\rho c)_{w}$ of the domain, while the thermal conductivity has been kept equal to the average value, since it has already been validated in (Alongi et al., 2017a). The values adopted in this analysis are $1511 \mathrm{~kJ} /\left(\mathrm{m}^{3} \cdot \mathrm{K}\right)$ (low capacity) and 2017 $\mathrm{kJ} /\left(\mathrm{m}^{3} \cdot \mathrm{K}\right)$ (high capacity), while the average values previously considered are reported in Table 1.

Again, the results of two sets of simulations have been compared to the corresponding heat flux densities calculated through the fitting process using Eq. (12), in order to obtain the average, standard deviation and maximum errors. All the outcomes of this process are listed in Table 5 and have to be compared to the corresponding quantities reported in Table 4 .

A careful analysis of these results shows that the highest accuracy is achieved with heat capacity equal or greater than the average: the average error drops in almost all airflow conditions when the high $C$ is set for calculations. The same happens also for standard deviations and maximum errors. As an example, in Figure 7 the experimental and simulated variations of heat flux density are visually compared for the airtight condition and for $u=0.012 \mathrm{~m} / \mathrm{s}$. As expected, the increase in heat capacity moves the fluctuation forward in time, leading to a smaller phase shift with respect to the fitted experimental curves, even though the amplitude prediction gets worse.

Table 5: average, standard deviation and maximum error calculated for heat flux density, for every airflow velocity considered, at low and high heat capacity.

\begin{tabular}{|c|c|c|c|c|c|c|c|}
\hline \multicolumn{2}{|c|}{$u[\mathrm{~m} / \mathrm{s}]$} & 0 & 0.001 & 0.003 & 0.006 & 0.009 & 0.012 \\
\hline \multicolumn{8}{|c|}{ low capacity } \\
\hline \multirow{3}{*}{ 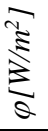 } & $\mu$ & 2.84 & 3.25 & 3.81 & 5.53 & 6.08 & 5.95 \\
\hline & $\sigma$ & 1.60 & 1.88 & 2.07 & 2.83 & 3.14 & 3.35 \\
\hline & $e_{\max }$ & 5.41 & 6.24 & 6.99 & 9.20 & 10.33 & 11.11 \\
\hline \multicolumn{8}{|c|}{ high capacity } \\
\hline \multirow{3}{*}{ 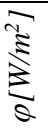 } & $\mu$ & 1.85 & 1.24 & 1.45 & 0.76 & 2.01 & 4.11 \\
\hline & $\sigma$ & 1.27 & 1.06 & 1.19 & 0.56 & 1.26 & 2.42 \\
\hline & $e_{\max }$ & 4.04 & 2.85 & 3.41 & 1.83 & 4.99 & 8.34 \\
\hline
\end{tabular}

These considerations could explain what has been previously noticed: the low accuracy of the numerical model in predicting the heat flux density at the surface might be due to inaccuracies in the material properties definition. Moreover, it has been noticed that a general improvement is obtained also in predicting temperature distributions, when the highest heat capacity is considered, even though these data are omitted for the sake of brevity. Hence, this analysis allows the Authors to infer that the actual sample heat capacity is higher than the average value from Table 1 .

Finally, the effects of the boundary conditions uncertainties on the simulated heat flux have neem evaluated. Going more in detail, the thermocouple accuracy of $0.15^{\circ} \mathrm{C}$ is either summed or subtracted to both outer and inner boundary conditions, in order to alternatively decrease or increase the almost null stationary temperature difference by $0.30{ }^{\circ} \mathrm{C}$. The new sets of boundary conditions have then been used to run new simulations and calculate the corresponding heat flux density variations.

The results of this new analysis are displayed in Figure 8, again for the airtight condition and for $u=0.012 \mathrm{~m} / \mathrm{s}$ only. In both cases, it appears clear how the heat flux density is largely influenced by the definition of the thermal boundary condition: the manipulation of their stationary components according to the thermocouples accuracy leads to instantaneous ranges of heat flux density fluctuations, that include the fitting of the measured values in most cases.

Therefore, these two sensitivity analyses focused on the heat flux density have shown that the inaccuracy of the numerical model observed at first (Table 4) might be due to a combined effect of several uncertainties, related to both the material heat capacity and the temperature 

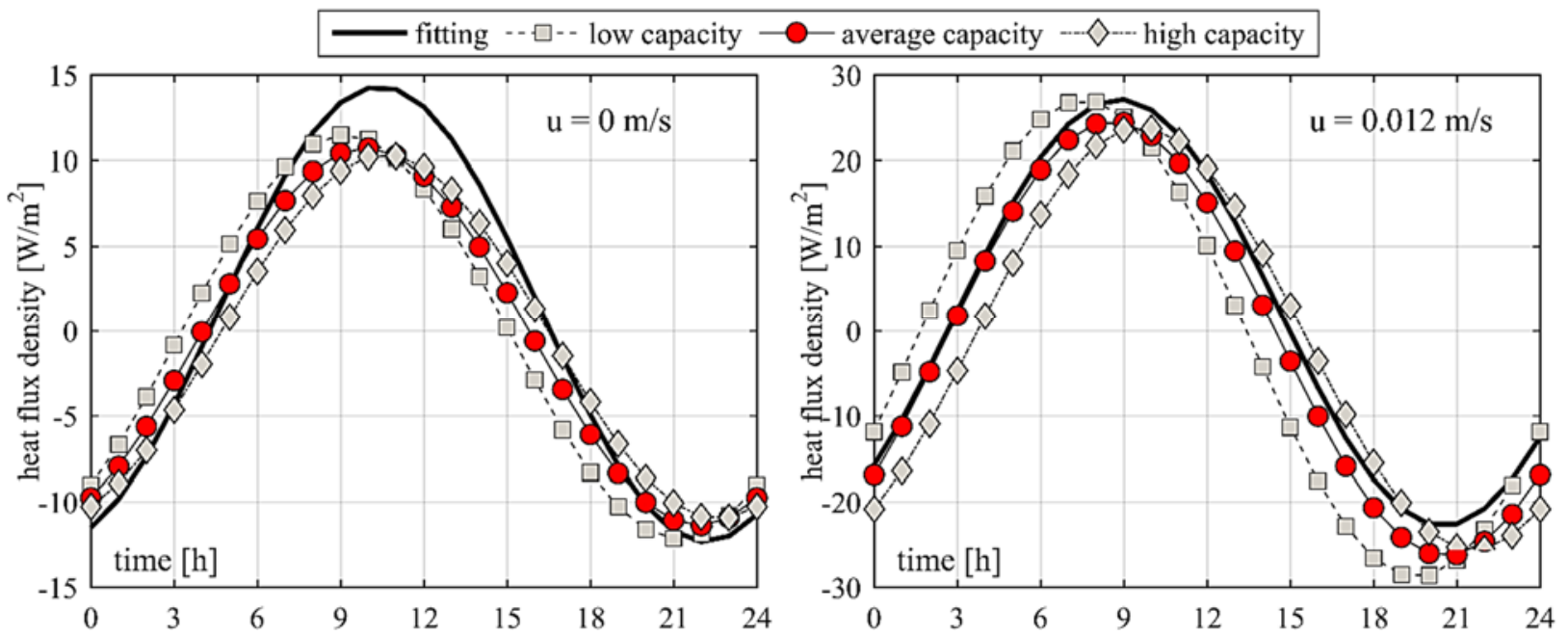

Figure 7: comparison between experimental data fitted through Eq.(11) and numerical results at various heat capacity levels, for airtight condition (left) and $u=0.012 \mathrm{~m} / \mathrm{s}$ (right).
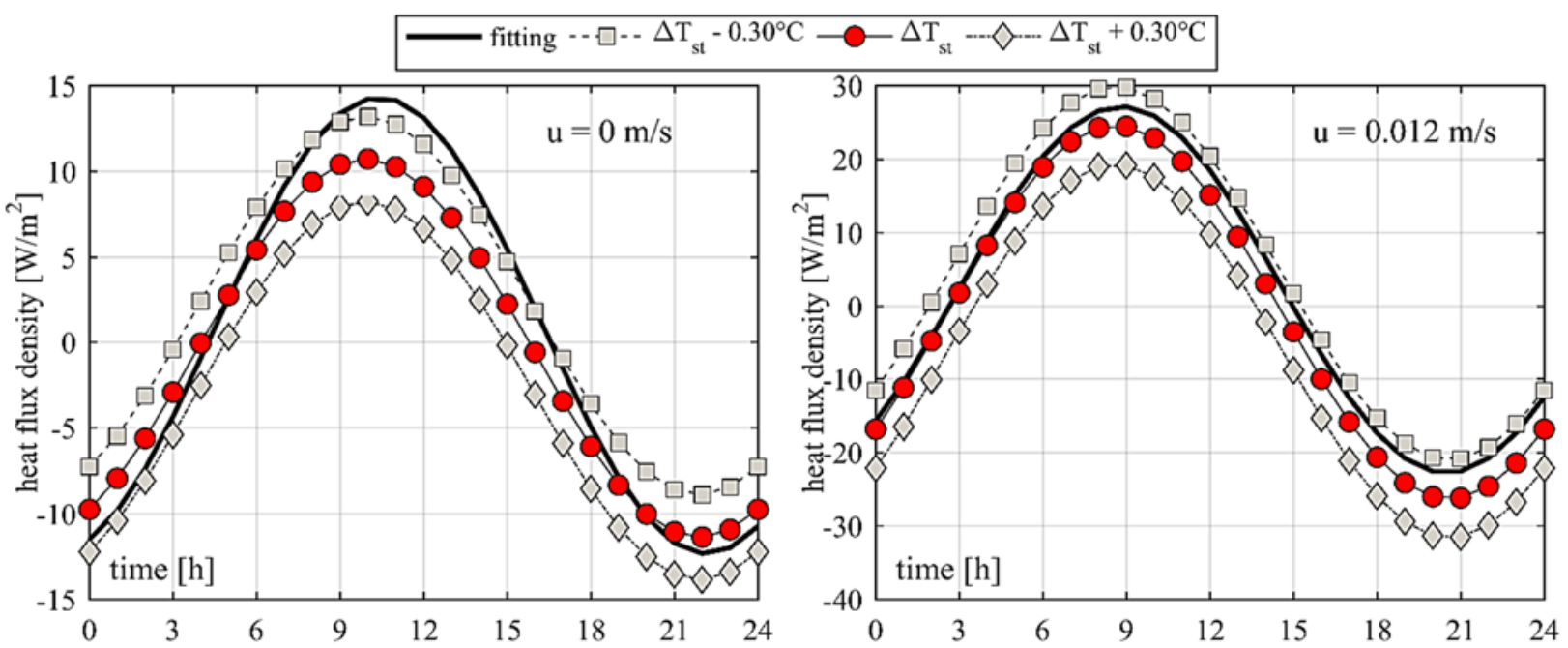

Figure 8: comparison between experimental data fitted through Eq.(11) and numerical results including the impact of the thermocouples accuracy on boundary conditions, for airtight condition (left) and $u=0.012 \mathrm{~m} / \mathrm{s}$ (right).

boundary conditions, and not to any intrinsic deficiency of the numerical model under discussion.

\section{Conclusions}

In this work, a numerical model aimed at the dynamic simulation of Breathing Wall components has been presented and validated against experimental data obtained from a series of tests performed on a no-fines concrete sample using the DAVTB facility. When simulation results are compared to the corresponding measured data:

- a good performance is observed in temperature distribution prediction, i.e. the average error is in the range $0.07^{\circ} \mathrm{C} \div 0.10^{\circ} \mathrm{C}$, while the highest standard deviation is $0.07{ }^{\circ} \mathrm{C}$. These results are then comparable with the calibrated thermocouple accuracy considered $\left(0.15^{\circ} \mathrm{C}\right)$;

- the Finite Difference Model is less effective in predicting the heat flux density at the edge of the domain. The average error goes from $1.90 \mathrm{~W} / \mathrm{m}^{2}$ to $2.62 \mathrm{~W} / \mathrm{m}^{2}$ (with a standard deviation around $1.25 \mathrm{~W} / \mathrm{m}^{2}$ ), which is significant if compared to the measured heat flux density fluctuation (that lies in the range $12.5 \mathrm{~W} / \mathrm{m}^{2} \div 25 \mathrm{~W} / \mathrm{m}^{2}$ ).

The sensitivity analysis to the spatial and temporal grid shows that:

- the space discretizations considered $(0.5 \mathrm{~mm}, 1 \mathrm{~mm}$ and $2 \mathrm{~mm}$ has no significant effect on the ability of the numerical model to predict both temperature distribution and heat flux density;

- the time discretization shows a greater effect on the numerical model accuracy in predicting temperature distribution. Actually both average and standard deviation of errors decrease when the simulation timestep is reduced from $3600 \mathrm{~s}$ to $900 \mathrm{~s}$. However, even the worst results are within the accuracy of the temperature probes.

It is also shown that the observed discrepancy between numerically simulated and measured heat flux density is compatible with both:

- a heat capacity for the material higher than average, suggesting that the actual heat capacity of the no-fines concrete sample tested might be higher than what initially considered; 
- the uncertainty in the surface temperatures boundary conditions imposed to the model, deriving from the thermocouple accuracy.

Therefore, all outcomes demonstrate the reliability of the implicit Finite Difference Method presented in this paper. The future development of this work might be focused first on the assessment of the surface heat transfer coefficients for Breathing Wall components inside the DAVTB facility, which will then be used to validate the Finite Difference Model under third type conditions; then, multilayer samples will be studied to collect the experimental data needed to validate also this feature of the numerical model. Finally, the Finite Difference numerical model of the Breathing Wall will be linked to a Building Energy Simulation tool, allowing to investigate the best strategies to integrate such technology in the building envelope and ventilation system.

\section{Nomenclature}

$\begin{array}{ll}c & \text { specific thermal capacity }[\mathrm{J} /(\mathrm{kg} \cdot \mathrm{K})] \\ C & \text { thermal capacity }\left[\mathrm{J} /\left(\mathrm{m}^{3} \cdot \mathrm{K}\right)\right] \\ e & \text { error }\left[{ }^{\circ} \mathrm{C}\right] /\left[\mathrm{W} / \mathrm{m}^{2}\right] \\ i & \text { space node index }[-] \\ j & \text { time node index }[-] \\ L & \text { size of the sample/domain }[\mathrm{m}] \\ M & \text { maximum time node index }[-] \\ N & \text { maximum space node index }[-] \\ T & \text { temperature }\left[{ }^{\circ} \mathrm{C}\right] \\ t & \text { time coordinate }[\mathrm{s}] \\ u & \text { airflow velocity }[\mathrm{m} / \mathrm{s}] \\ X & \text { space coordinate }[\mathrm{m}]\end{array}$

Greek symbols

$\begin{array}{ll}\alpha & \text { thermal diffusivity }\left[\mathrm{m}^{2} / \mathrm{s}\right] \\ \beta & \text { advective term coefficient }[\mathrm{m} / \mathrm{s}] \\ \varepsilon & \text { porosity }[-] \\ \varphi & \text { heat flux density }\left[\mathrm{W} / \mathrm{m}^{2}\right] \\ \lambda & \text { thermal conductivity }[\mathrm{W} /(\mathrm{m} \cdot \mathrm{K})] \\ \mu & \text { average error }\left[{ }^{\circ} \mathrm{C}\right] /\left[\mathrm{W} / \mathrm{m}^{2}\right] \\ \rho & \text { density }\left[\mathrm{kg} / \mathrm{m}^{3}\right] \\ \sigma & \text { standard deviation of errors }\left[{ }^{\circ} \mathrm{C}\right] /\left[\mathrm{W} / \mathrm{m}^{2}\right] \\ \psi & \text { angular phase }[\mathrm{rd}] \\ \omega & \text { angular frequency }[\mathrm{rd} / \mathrm{s}] \\ \text { Subscripts } & \\ f & \text { quantity referred to the fluid phase } \\ \text { max } & \text { maximum } \\ w & \text { quantity referred to the porous material }\end{array}$

\section{References}

Alongi A., Mazzarella L. (2015). The Dual Air Vented Thermal Box: a laboratory apparatus to test air permeable building envelope technologies. Energy Procedia (78), 1543-1548.

Alongi A., Angelotti A., Mazzarella L. (2017a). Experimental investigation of the steady state behaviour of Breathing Walls by means of a novel laboratory apparatus. Building and Environment (123), 415-426.
Alongi A., Angelotti A., Mazzarella L. (2017b). Analytical modelling of Breathing Walls: experimental verification by means of the Dual Air Vented Thermal Box lab facility. Energy Procedia (140), 36-47.

Ascione F., Bianco N., De Stasio C., Mauro G.M., Vanoli G.P. (2015). Dynamic insulation of the building envelope: numerical modeling under transient conditions and coupling with nocturnal free cooling, Applied Thermal Engineering (84), 1-14.

Craig S. and Grinham J. (2017). Breathing walls: The design of porous materials for heat exchange and decentralized ventilation. Energy and Buildings (149), 246-259.

Di Giuseppe E., D’Orazio M., Di Perna C. (2015). Thermal and filtration performance assessment of a dynamic insulation system. Energy Procedia (78), 513-518.

Dimoudi A., Androutsopoulos A., Lykoudis S. (2004). Experimental work on a linked, dynamic and ventilated wall component. Energy and Building (36), 443-453.

Gan G. (2000). Numerical evaluation of thermal comfort in rooms with dynamic insulation. Building and Environment (35), 445- 453.

Imbabi M.S. (2004). New developments in the science and art of Breathing Walls. Proceedings from WREC 2004: World Renewable Energy Congress VIII. Denver (CO), 1-8 September 2004.

Imbabi M.S. (2006). Modular breathing panels for energy efficient, healthy building construction. Renewable Energy (31), 729-738.

Imbabi M.S. (2012). A passive-active dynamic insulation system for all climates. International Journal of Sustainable Built Environment (1), 247-258.

Krarti M. (1994). Effect of air flow on heat transfer in walls. Journal of Solar Energy Engineering (116), 3542.

Taylor B.J., Cawthorne D.A., Imbabi M.S. (1996). Analytical investigation of the steady-state behaviour of dynamic and diffusive building envelopes. Building and Environment (31), 519-525.

Taylor B.J. and Imbabi M.S. (1998). The application of dynamic insulation in buildings. Renewable Energy (15), 377-382.

Taylor B.J., Webster R., Imbabi M.S. (1999). The building envelope as an air filter. Building and Environment (34), 353-361.

Wong J.M., Glasser F.P., Imbabi M.S. (2007), Evaluation of thermal conductivity in air permeable concrete for dynamic breathing wall construction. Cement \& Concrete Composites (29) 647-655. 